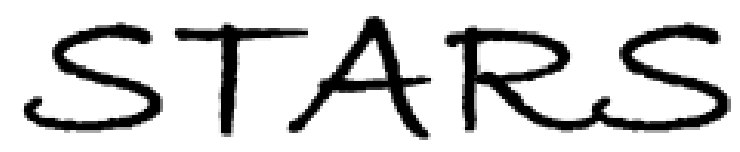

University of Central Florida

STARS

$1-1-2011$

\title{
Impact of forward bias injection on minority carrier transport in $\mathrm{p}$ - type $\mathrm{ZnO}$ nanowires
}

\author{
C. Schwarz \\ University of Central Florida \\ E. Flitsiyan \\ University of Central Florida \\ L. Chernyak \\ University of Central Florida \\ V. Casian \\ R. Schneck
}

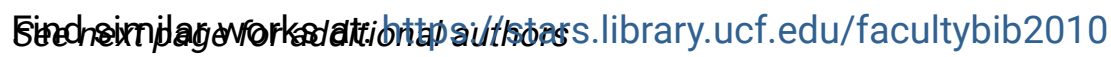

University of Central Florida Libraries http://library.ucf.edu

This Article is brought to you for free and open access by the Faculty Bibliography at STARS. It has been accepted for inclusion in Faculty Bibliography 2010 s by an authorized administrator of STARS. For more information, please contactSTARS@ucf.edu.

\section{Recommended Citation}

Schwarz, C.; Flitsiyan, E.; Chernyak, L.; Casian, V.; Schneck, R.; Dashevsky, Z.; Chu, S.; and Liu, J. L., "Impact of forward bias injection on minority carrier transport in p-type ZnO nanowires" (2011). Faculty

Bibliography 2010s. 1876.

https://stars.library.ucf.edu/facultybib2010/1876

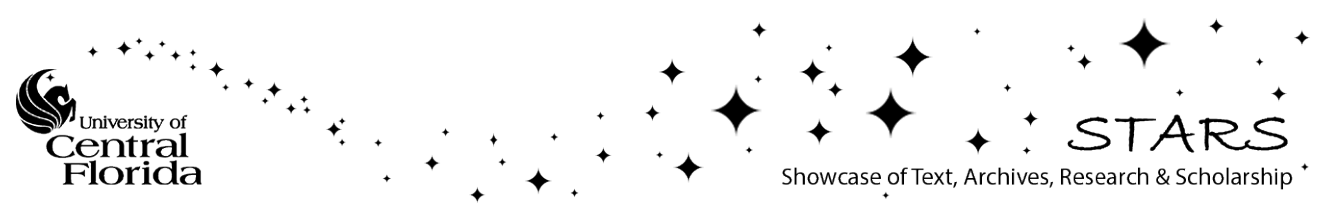


Authors

C. Schwarz, E. Flitsiyan, L. Chernyak, V. Casian, R. Schneck, Z. Dashevsky, S. Chu, and J. L. Liu 


\section{Impact of forward bias injection on minority carrier transport in p-type ZnO nanowires}

Cite as: J. Appl. Phys. 110, 056108 (2011); https://doi.org/10.1063/1.3633224

Submitted: 24 June 2011 . Accepted: 03 August 2011 . Published Online: 09 September 2011

C. Schwarz, E. Flitsiyan, L. Chernyak, V. Casian, R. Schneck, Z. Dashevsky, S. Chu, and J. L. Liu

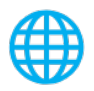

\section{ARTICLES YOU MAY BE INTERESTED IN}

Minority carrier transport in $\mathrm{p}-\mathrm{ZnO}$ nanowires

Journal of Applied Physics 109, 016107 (2011); https://doi.org/10.1063/1.3530732

Diameter dependence of the minority carrier diffusion length in individual ZnO nanowires Applied Physics Letters 96, 253115 (2010); https://doi.org/10.1063/1.3456390

Electron-beam-induced current and cathodoluminescence studies of thermally activated increase for carrier diffusion length and lifetime in $n$-type $\mathrm{ZnO}$

Applied Physics Letters 87, 162103 (2005); https://doi.org/10.1063/1.2106001

\section{Applied Physics Reviews} Now accepting original research

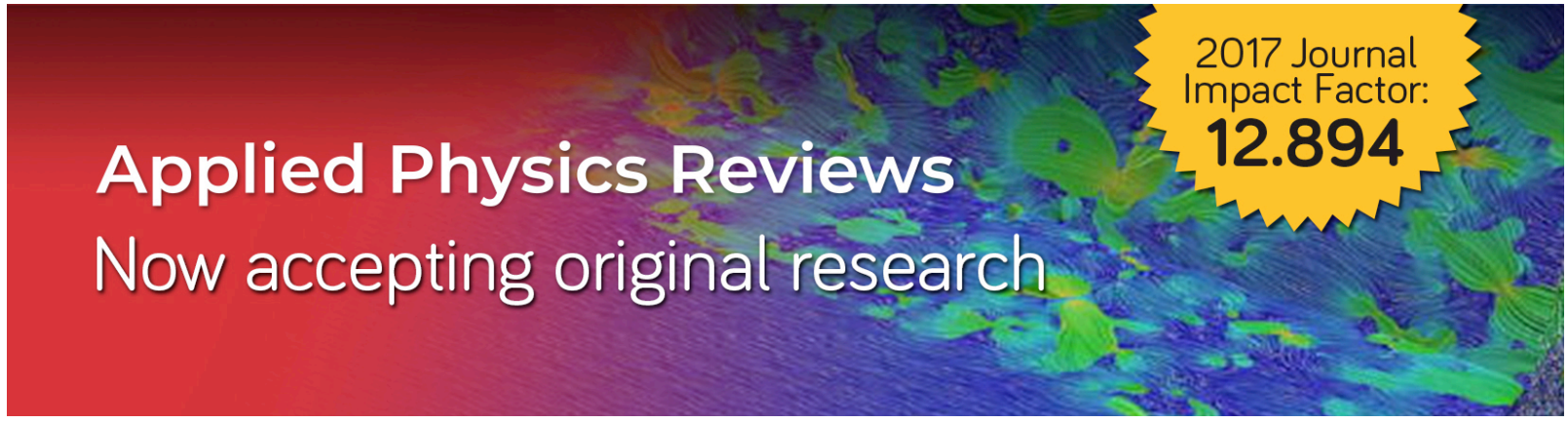




\title{
Impact of forward bias injection on minority carrier transport in p-type $\mathrm{ZnO}$ nanowires
}

\author{
C. Schwarz, ${ }^{1}$ E. Flitsiyan, ${ }^{1}$ L. Chernyak, ${ }^{1, a)}$ V. Casian, ${ }^{2}$ R. Schneck, ${ }^{2}$ Z. Dashevsky, ${ }^{2}$ \\ S. Chu, ${ }^{3}$ and J. L. Liu ${ }^{3}$ \\ ${ }^{1}$ Department of Physics, University of Central Florida, Orlando, Florida 32816-2385, USA \\ ${ }^{2}$ Department of Materials Engineering, Ben-Gurion University, Beer-Sheva 84105, Israel \\ ${ }^{3}$ Department of Electrical Engineering, University of California, Riverside, California 92521, USA
}

(Received 24 June 2011; accepted 3 August 2011; published online 9 September 2011)

\begin{abstract}
Minority carrier diffusion length in $p$-type $\mathrm{Sb}$-doped $\mathrm{ZnO}$ nanowires was measured as a function of temperature and forward bias injection duration. The minority carrier diffusion length displays a thermally activated length increase with the energy of $144 \pm 5 \mathrm{meV}$. The forward bias injection exhibits an increase in diffusion length with the activation energy of $217 \pm 20 \mathrm{meV}$, indicating the possible involvement of a $\mathrm{Sb}_{\mathrm{Zn}}-2 \mathrm{~V}_{\mathrm{Zn}}$ acceptor complex. (C) 2011 American Institute of Physics. [doi:10.1063/1.3633224]
\end{abstract}

ZnO's fundamental properties, such as its wide-band gap $(3.37 \mathrm{eV})$, high breakdown strength, and large exciton binding energy $(60 \mathrm{meV})$ have generated much interest in this material for use in optoelectronic devices. Coupled with the recent opportunity for $\mathrm{ZnO}$ material to be manufactured in the shape of nanowires, greater prospects continue to emerge for $\mathrm{ZnO}$ in the field of solid-state electronics. The large surface area of nanowires in combination with the biosafe characteristics of $\mathrm{ZnO}$ makes them attractive candidates for gas, chemical sensing, and biomedical applications. ${ }^{10}$ However, achieving good $p$-type conductivity has been a major hurdle for developing $\mathrm{ZnO}$ p-n junctions due to the high ionization energies of its potential acceptors. Recent advances in $p$-type doping using larger radii atoms, such as antimony $(\mathrm{Sb})$, have produced $\mathrm{ZnO}$ homojunctions with good electrical and optical properties. ${ }^{14}$ Studying the diffusion length of minority carriers in $\mathrm{ZnO}$ is subsequently important because this property defines the performance of $p-n$ junctions and bipolar devices.

Leading up to this study on forward bias injection in $\mathrm{ZnO}$ nanowires, the increase in minority carrier diffusion length, $L$, through temperature and electron beam irradiation effects have been explored and analyzed both in $p$ - and $n$-type $\mathrm{GaN}$ and $\mathrm{ZnO}^{2-4,12}$ Due to advances in $p$-type doping, ZnO's unique properties and its advantages over $\mathrm{GaN}$, our focus has been recently shifted to exploring the transport properties in $\mathrm{ZnO}$. Studies show that an increase in $L$ can be induced by temperature and electron beam irradiation for epitaxial $p$-type $\mathrm{ZnO}$ doped with $\mathrm{Sb}^{2,3}$ Minority carrier transport has also been recently studied in $p$-type $\mathrm{Sb}$ doped $\mathrm{ZnO}$ nanowires subjected to electron beam irradiation. ${ }^{6}$ It is also of interest that an increase in minority carrier diffusion length, similar to the electron beam irradiation effect, can be witnessed in $p$-type $\mathrm{GaN}$ and $\mathrm{ZnO}$ through forward bias electron injection in the epitaxial p-n homo-junctions. ${ }^{1,13}$ In this paper, we report the impact of forward bias electron injection on minority carrier diffusion length in $p$-type Sb-doped $\mathrm{ZnO}$ nanowires at variable temperatures.

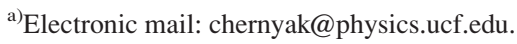

Experiments reported in this work were carried out on $p$-type Sb-doped nanowire layers. The sample consists of a very thin $\mathrm{MgO}$ buffer layer and a $2 \mu \mathrm{m}$-thick, undoped $n$-type $\mathrm{ZnO}$ epitaxial film grown on a $\mathrm{Si}(100)$ substrate by molecular beam epitaxy (MBE) under a growth temperature of $450^{\circ} \mathrm{C}$. The sample was then transferred to a Thermal Scientific quartz tube chemical vapor deposition (CVD), in which the nanowires were grown. During the nanowire growth, the temperature was kept at $600{ }^{\circ} \mathrm{C}$. The result was a $p$-type $\mathrm{ZnO}$ nanowire layer measured to be about $5 \mu \mathrm{m}$ thick. The top surface of nanowire layer was then covered with a silver paste for contacting purposes.

We used the electron beam-induced current (EBIC) technique to extract the minority carrier diffusion length of $p$-type $\mathrm{ZnO}$ nanowires. The EBIC technique is dependent on the nonequilibrium carriers generated in semiconductors under the influence of electron beam irradiation and their resulting separation by the built in field of the p-n junction or Schottky diode. As majority carriers are swept away from the region of the p-n junction's built-in field localization, the minority ones instead build up and strengthen the magnitude of the overall electric current (i.e., EBIC). By measuring the EBIC current dependence on the distance from the p-n junction and with the use of Eq. (1), the minority carrier diffusion length, $L$, can then be extracted from the EBIC line scan. ${ }^{4}$ Using this technique, we measure the minority carrier diffusion length in $\mathrm{Sb}$ doped $\mathrm{ZnO}$ nanowire layers (cleaved perpendicular to the growth plane) under various temperatures and with forward bias injection. The EBIC signal was recorded within the nanowire layer, beginning from the $n-\mathrm{ZnO} / p-\mathrm{ZnO}$ interface and continuing to the $p-\mathrm{ZnO}$ nanowire region towards the surface, on a sample cleaved perpendicular to the plane of growth (Fig. 1). The EBIC technique depends explicitly on the space charge region of a sample where the internal electric field exists to separate the carriers. In Fig. 1, one can see the superimposed EBIC line-scan signal following a path of exponential decay in the $p$-type $\mathrm{ZnO}$ nanowire region.

All experiments were conducted in situ in a Philips XL30 scanning electron microscope (SEM) under an accelerating voltage of $20 \mathrm{kV}$. A forward bias was applied to the 


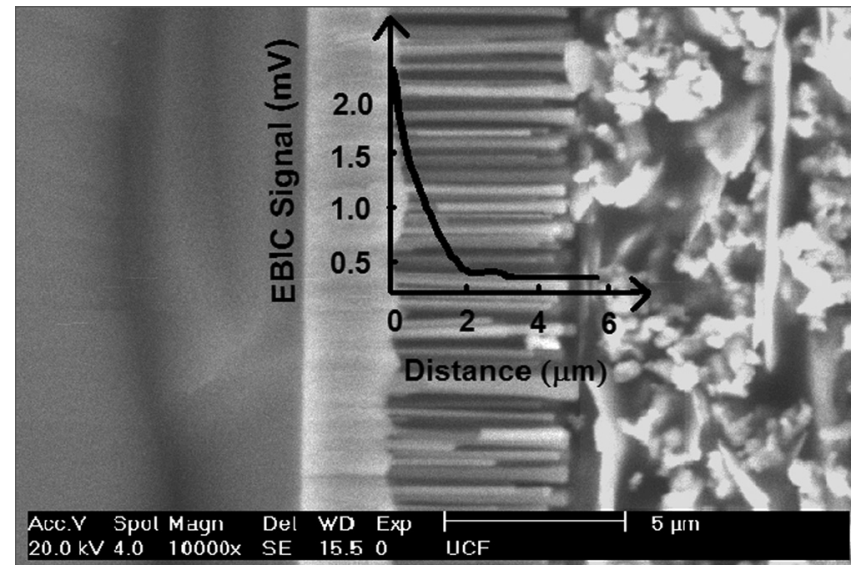

FIG. 1. Secondary electron image of the samples cross section taken perpendicular to the growth plane. From left to right: silicon substrate; $\mathrm{n}-\mathrm{ZnO}$ film; p-ZnO (nanowire layer); and Ag-epoxy layer (used for a contact). EBIC measurements were taken from the $\mathrm{n}-\mathrm{ZnO}$ film $/ \mathrm{p}-\mathrm{ZnO}$ nanowire interface. Superimposed: Exponential decay of EBIC signal versus the beam-tobarrier distance.

p-n junction between the $p$-type $\mathrm{ZnO}$ nanowires and the $n$-type $\mathrm{ZnO}$ film using a Hewlett-Packard 4145A semiconductor parameter analyzer resulting in a current of about 5 $\mathrm{mA}$. After the initial line-scan (24 s), EBIC measurement, and extraction of diffusion length $L$, the forward bias injection was continued by applying $12 \mathrm{~V}$ for up to $600 \mathrm{~s}$ in $100 \mathrm{~s}$ increments. This was repeated at temperatures adjusted to $25,45,55$, and $75^{\circ} \mathrm{C}$ using an external temperature controller (Gatan). Before measurements were conducted, the sample was freshly cleaved and placed in a vacuum chamber at $2 \times 10^{-6}$ mBar. Because the electron beam excitation during the measurement period ( $24 \mathrm{~s}$ ) was much shorter than the duration of the forward bias injection (hundreds of seconds), the contribution of SEM beam to the electron injection effect as well as to desorption of oxygen and other species (if any) from the nanowires was negligible. A new region was also selected at each temperature and the electron beam was turned off while applying forward bias. Areas for measurements were selected based on absence of visible particles in the region under investigation, as seen from the SEM picture (Fig. 1).

Before performing the experiments on forward bias electron injection in $p$ - $\mathrm{ZnO}$ nanowires, we first determined the impact of temperature on the minority carrier diffusion length of the sample. The exponential decay of the EBIC signal within the nanowire layer versus distance from the $p-n$ junction interface is fitted to Eq. (1)

$$
I=A d^{\alpha} \exp \left(-\frac{d}{L}\right),
$$

where $I$ is the EBIC signal, $A$ is a constant, $d$ is beam-tojunction distance, and $L$ is the diffusion length. The coefficient, $\alpha$, responsible for surface recombination, was taken to equal $-1 / 2$. $^{5}$ The relationship between diffusion length, $L$, and temperature is displayed in Fig. 2. One can see from this figure that the value of $L$ increases exponentially with temperature increase; this is fitted to Eq. (2)

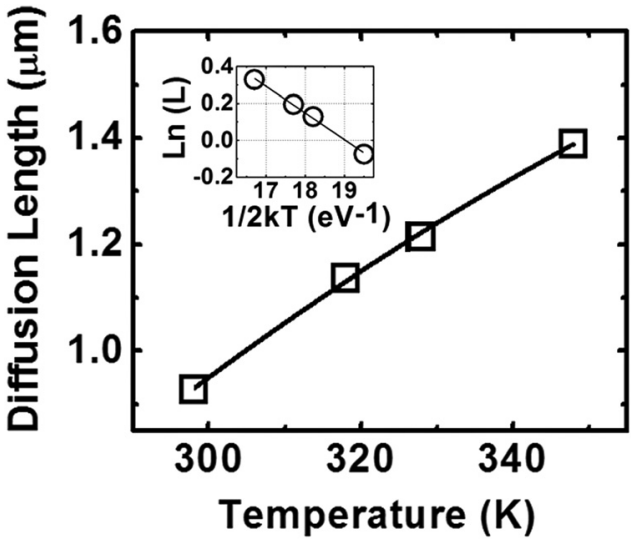

FIG. 2. Dependence of diffusion length on temperature in the nanowire p- $\mathrm{ZnO}$ region. Inset: Arrhenius plot for $L$ vs. $T$ dependence resulting in a value for activation energy of $144 \pm 5 \mathrm{meV}$.

$$
L=L_{0} \sqrt{\exp \left(-E_{a} / k T\right)}
$$

where $L_{0}$ is a scaling factor, $k$ is Boltzmann constant, and $E_{a}$ is the thermal activation energy found to be $144 \pm 5 \mathrm{meV}$ (obtained from the Arrhenius plot in the inset of Fig. 2). The latter parameter likely represents carrier delocalization energy and determines the increase of the diffusion length due to the reduction in recombination efficiency. ${ }^{3}$ Previous photo- and cathodo-luminescence studies on $p$-type $\mathrm{ZnO}$ have shown that the recombination route of nonequilibrium carriers involves transitions to a deep, neutral acceptor level $\left(\mathrm{e}, \mathrm{A}^{0}\right){ }^{9,11}$ Increasing the temperature of the sample leads to a larger ionization fraction of the acceptors while inhibiting the recombination rate by reducing the concentration of $\mathrm{A}^{0}$.

The application of a forward bias to the $p-n$ junction of the sample also resulted in an increase in the minority carrier diffusion length (Fig. 3). However, as the temperature was raised under forward bias, a relative reduction in the diffusion length growth rate can also be observed (Fig. 3). This forward bias injection-induced increase of $L$ suggests that it comes from a similar origin to that of the electron beam irradiation-induced increase. ${ }^{6}$ The effect of electron irradiation on the diffusion length can be attributed to the trapping of non-equilibrium electrons on the neutral acceptor levels $\left(\mathrm{A}^{0}+\mathrm{e}^{-} \rightarrow \mathrm{A}^{-}\right)$.

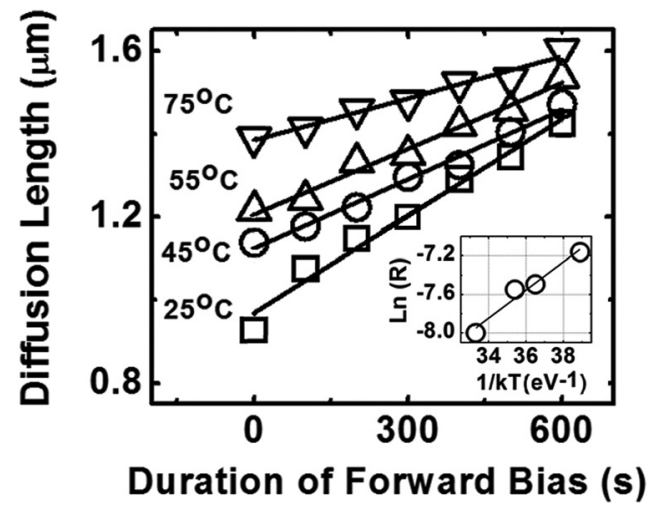

FIG. 3. Linear dependence of the diffusion length on forward bias duration under increasing temperature. The rates, $R$, of increase of $L$ for each temperature is obtained from the slope. Inset: Arrhenius plot for $R$ vs. $T$ dependence resulting in the activation energy of $217 \pm 20 \mathrm{meV}$. 
When the non-equilibrium electron-hole pairs recombine, they involve the levels of neutral acceptors located deep in the band gap. Electron trapping on neutral acceptor levels prohibits the pathway of recombination via these levels and leads to an increase of non-equilibrium minority carrier (electron) lifetime in the conduction band and, in turn, carrier diffusion length. As the excitation proceeds, the concentration of neutral levels decreases, due to electron trapping on them, while the diffusion length steadily rises. Since the presence of $\mathrm{Sb}$ has been shown to induce acceptor levels in the band gap far from the valence band edge, it is likely that applying forward bias results in similar effects. This is because the electrons injected into $p-\mathrm{ZnO}$ become trapped on the Sb-related levels preventing further recombination via these levels and subsequently resulting in the increase of $L .{ }^{1}$ The dependence of forward bias injection and temperature on the rate R, can be quantified by Eq. (3) (Ref. 7)

$$
R=R_{0} \exp \left(\frac{E_{f b i}}{k T}\right) \exp \left(-\frac{E_{a}}{2 k T}\right)
$$

where $R_{0}$ is a scaling factor; $\mathrm{k}$ is Boltzmann constant; $E_{a}$ is the thermal activation energy determined from the abovementioned temperature-dependent $L$ measurements $(\sim 144$ $\mathrm{meV}) ; E_{f b i}$ is the activation energy for the forward bias injection-induced effect. Accounting for rates, $R$, at various temperatures, which can be obtained from the dependences of $L$ on forward bias injection duration in Fig. 3, $E_{f b i}$ can be calculated from (3) using a linear fitting of $\ln (R)$ vs. $1 / k T$, as shown in the inset of Fig. 3. We found $E_{f b i}$ to be $217 \pm 20$ $\mathrm{meV}$. This is in good agreement with our previous work on p-type $\mathrm{ZnO}$ nanowires, where the impact of electron beam irradiation on minority carrier transport was studied. ${ }^{6}$

It has been theorized by Limpijumnong et al. that the role of acceptors in size-mismatched Sb-impurity doped $\mathrm{ZnO}$ is accomplished by a $\mathrm{Sb}_{\mathrm{Zn}}-2 \mathrm{~V}_{\mathrm{Zn}}$ complex, which is predicted to have an ionization energy of $160 \mathrm{meV}{ }^{8}$ While our value for activation energy $(217 \mathrm{meV})$ is somewhat larger than the predicted value $(160 \mathrm{meV})$, we can successfully rule out other $\mathrm{Sb}$-related defects such as $\mathrm{Sb}_{\mathrm{o}}$ substitutional defects as well as the single vacancy $\mathrm{Sb}_{\mathrm{Zn}}-\mathrm{V}_{\mathrm{Zn}}$ complex, due to their ionization energies being about an order of magnitude larger than our value. ${ }^{8}$ It has also been shown that in
Sb-doped $\mathrm{ZnO}$, acceptor activation energy can vary from about $135 \mathrm{meV}\left(1.3 \times 10^{18} \mathrm{~cm}^{-3}\right)$ to $212 \mathrm{meV}\left(1.3 \times 10^{17}\right.$ $\mathrm{cm}^{-3}$; comparable to the case of $\mathrm{ZnO}$ nanowires used in this study) depending on its concentration of majority carriers. ${ }^{9}$

In conclusion, forward bias injection leads to an increase in minority carrier diffusion length in $p$-type $\mathrm{Sb}$-doped $\mathrm{ZnO}$ nanowires. Temperature dependent EBIC measurements allow for the estimation of the activation energy of this increase. Using the experimentally obtained values for the activation energy and comparing to known values, the $\mathrm{Sb}_{\mathrm{Zn}}-2 \mathrm{~V}_{\mathrm{Zn}}$ acceptor-complex was identified as a possible origin for carrier trapping on the deep Sb-related acceptor levels.

The authors would like to thank the National Science Foundation (ECCS Grant No. 0900971) and the U.S.-Israel Binational Science Foundation (Grant No. 2008328) for financial support.

${ }^{1}$ O. Lopatiuk-Tirpak, L. Chernyak, L. J. Mandalapu, Z. Yang, J. L. Liu, K. Gartsman, Y. Feldman, and Z. Dashevsky, Appl. Phys. Lett. 89, 142114 (2006).

${ }^{2}$ L. Chernyak, C. Schwarz, E. Flitsiyan, S. Chu, J. L.Liu, and K. Gartsman, Appl. Phys. Lett. 92, 102106 (2008).

${ }^{3}$ O. Lopatiuk-Tirpak, F. X. Xiu, J. L. Liu, S. Jang, F. Ren, S. J. Pearton, K. Gartsman, Y. Feldman, A. Osinsky, P. Chow, and L. Chernyak, J. Appl. Phys. 100, 086101 (2006).

${ }^{4}$ L. Chernyak, A. Osinsky, H. Temkin, J. W. Yang, Q. Chen, and M. A. Khan, Appl. Phys. Lett. 69, 2531 (1996).

${ }^{5}$ Y. Lin, E. Flitsyian, L. Chernyak, T. Malinauskas, R. Aleksiejunas, K. Jarasiunas, W. Lim, S. J. Pearton, and K. Gartsman, Appl. Phys. Lett. 95, 092101 (2009).

${ }^{6}$ Y. Lin, M. Shatkhin, E. Flitsyian, L. Chernyak, Z. Dashevsky, S. Chu, and J. L. Liu, J. Appl. Phys. 109, 016107 (2011).

${ }^{7}$ O. Lopatiuk, L. Chernyak, A. Osinsky, and J. Q. Xie, Appl. Phys. Lett. 87, 214110 (2005).

${ }^{8}$ S. Limpijumnong, S. B. Zhang, S. H. Wei, and C. H. Park, Phys. Rev. Lett. 92, 155504 (2004).

${ }^{9}$ O. Lopatiuk-Tirpak, W. V. Schoenfeld, L. Chernyak, F. X. Xiu, J. L. Liu, S. Jang, F. Ren, S. J. Pearton, A. Osinsky, and P. Chow, Appl. Phys. Lett. 88, 202110 (2006).

${ }^{10}$ Y. W. Heo, D. P. Norton, L. C. Tien, Y. Kwon, B. S. Kang, F. Ren, S. J. Pearton, J. R. LaRoche, Mater. Sci. Eng. R 47, 1 (2004).

${ }^{11}$ F. X. Xiu, Z. Yang, L. J. Mandalapu, D. T. Zhao, J. L. Liu, and W. P. Beyermann, Appl. Phys. Lett. 87, 152101 (2005).

${ }^{12}$ L. Chernyak, A. Osinsky, V. Fuflyigin, and E. F. Schubert, Appl. Phys. Lett. 77, 875 (2000).

${ }^{13}$ L. Chernyak, G. Nootz, and A. Osinsky, Electronics Letters. 37, 922 (2001).

${ }^{14}$ L. Chernyak, A. Osinsky, and A. Schulte, Solid-State Electron. 45, 1687 (2001). 